\title{
Almost Phase Retrieval with Few Intensity Measurements
}

\author{
Wei Zhong \\ Department of Mathematics and LPMC, Nankai University, Tianjin 300071, China \\ Correspondence should be addressed to Wei Zhong; qmzw@mail.nankai.edu.cn
}

Received 10 June 2014; Accepted 22 August 2014; Published 25 September 2014

Academic Editor: Hang Xu

Copyright (C) 2014 Wei Zhong. This is an open access article distributed under the Creative Commons Attribution License, which permits unrestricted use, distribution, and reproduction in any medium, provided the original work is properly cited.

In signal processing, the phases of the measurements are often unknown. To recover a signal of length $N$, Balan, Casazza, and Edidin showed that it suffices to know at least $2 N-1$ intensity measurements. We give another characterization of frames which give phase retrieval for almost all signals with only $N+1$ intensity measurements. We provide a method to test if a frame has this property or not. With our method, we can construct tight frames or modify any frames such that they give almost phase retrieval. Numerical results show that frames with this property can recover signals with high probability.

\section{Introduction}

Given a set of vectors $\left\{\varphi_{k}\right\}_{1 \leq k \leq M} \subset \mathbb{K}^{N}(\mathbb{K}=\mathbb{R}$ or $\mathbb{C})$, the phase retrieval problem is to recover a signal from $\left\{\left|\left\langle x, \varphi_{k}\right\rangle\right|\right\}_{1 \leq k \leq M}$ which is called intensity measurements. Phase retrieval is a key step in speech processing [1], X-ray crystallography [2], and so forth. Since frames are redundant systems of vectors in a Hilbert space, it is possible to reconstruct signals with suitable frames.

First, we introduce the definition of phase retrieval.

Definition 1 (see [3, Definition 2.4]). One calls that a set of vectors $\left\{\varphi_{k}\right\}_{1 \leq k \leq M}$ in $\mathbb{K}^{N}$ gives phase retrieval if for all $x, y \in$ $\mathbb{K}^{N}$ satisfying $\left|\left\langle x, \varphi_{k}\right\rangle\right|=\left|\left\langle y, \varphi_{k}\right\rangle\right|$, for all $k=1,2, \ldots, M$, there exists a constant $c \in \mathbb{K}$ with $|c|=1$ such that $x=c y$.

In fact, this definition is equivalent to the following.

Definition 2. One calls that a set of vectors $\left\{\varphi_{k}\right\}_{1 \leq k \leq M}$ in $\mathbb{K}^{N}$ gives phase retrieval if for all $x, y \in \mathbb{K}^{N}$ satisfying

$$
\left\langle x, \varphi_{k}\right\rangle=c_{k}\left\langle y, \varphi_{k}\right\rangle, \quad 1 \leq k \leq M,
$$

where $c_{k} \in \mathbb{K}$ and $\left|c_{k}\right|=1$, one has $c_{1}=c_{2}=\cdots=c_{M}$.

Let $W$ be an $N \times M$ matrix of rank $N$. If the set of columns of $W$ gives phase retrieval, we also say that the matrix $W$ gives phase retrieval.
Balan et al. [4] studied the properties of such frames with minimal measurements. For the real frames, they gave the following result.

Proposition 3 (see [4, Theorem 2.2]). Let $\Phi=\left\{\varphi_{k}\right\}_{1 \leq k \leq M}$ be a frame for $\mathbb{R}^{N}$. If $M \geq 2 N-1$, then a generic frame $\Phi$ gives phase retrieval.

Here a generic frame $\Phi$ means a member of some nonempty Zariski open subset of the set of all $M$-element frames in $\mathbb{R}^{N}$. We refer to [5, Section 2.2] for details.

For the complex frame, a similar result also holds.

Proposition 4 (see [4, Theorem 2.2]). Let $\Phi=\left\{\varphi_{k}\right\}_{1 \leq k \leq M}$ be a frame for $\mathbb{C}^{N}$. If $M \geq 4 N-2$, then a generic frame $\Phi$ gives phase retrieval.

For $\mathbb{R}^{N}, M \geq 2 N-1$ is necessary. But for $\mathbb{C}^{N}$, Bandeira et al. conjectured the following.

Conjecture 5 (see [6]). Let $\Phi=\left\{\varphi_{k}\right\}_{1 \leq k \leq M}$ be a frame for $\mathbb{C}^{N}$, $N \geq 2$. Then the following holds.

(i) If $M<4 N-4$, then $\Phi$ does not give phase retrieval.

(ii) If $M \geq 4 N-4$ and $\Phi$ is a generic frame, then $\Phi$ gives phase retrieval. 
Then, Conca et al. [5] confirmed Conjecture 5(ii). But (i) is still an open problem.

Recently, people have studied more properties of such frames. Ohlsson and Eldar [7] studied the minimal measurements of sparse signals. If sparse signals contain noise, Eldar and Mendelson [8] also studied the minimal measurements and gave a recovery algorithm. By convex programming, Candès, Strohmer, and Vladislav gave a method which is called phaselift to recover a signal from its magnitude measurements. Then Gross et al. [9] and Alexeev et al. [10] developed other methods with phaselift. Chen et al. [11] estimated the error of phaselift. Schniter and Rangan [12] used compressed sensing to recover sparse signals via generalized approximate message passing.

If we do not require that $\Phi$ gives phase retrieval, for all $x \in \mathbb{K}^{N}$, we may need fewer measurements. Fickus et al. [13] studied when $\Phi$ gives phase retrieval for almost all vectors of $\mathbb{K}^{N}$. In this paper, we first introduce some preliminary results of frames which give phase retrieval. Then, we show our characterization and some properties of such frames. We give a method to test if a frame gives almost phase retrieval. Finally, we provide some examples to illustrate our results.

\section{Main Results}

First, we introduce some preliminary results.

A set of finitely many vectors $\Phi=\left\{\varphi_{k}\right\}_{1 \leq k \leq M}$ in $\mathbb{K}^{N}$ is called a frame if there are two positive constants $\alpha \leq \beta$ such that, for every $x \in \mathbb{K}^{N}$,

$$
\alpha\|x\|^{2} \leq \sum_{k=1}^{M}|\langle x, \varphi\rangle|^{2} \leq \beta\|x\|^{2} .
$$

A frame is said to be tight if $\alpha=\beta$ and Parseval if $\alpha=\beta=1$. If the right-hand side of (2) holds, it is said to be a Bessel sequence. Define the analysis and synthesis operators of a frame by

$$
\begin{array}{cc}
T: \mathbb{K}^{N} \longrightarrow l^{2}, & T(x)=\left\{\left\langle x, \varphi_{k}\right\rangle\right\}_{1 \leq k \leq M}, \\
T^{*}: l^{2} \longrightarrow \mathbb{K}^{N}, & T^{*}\left\{c_{k}\right\}_{1 \leq k \leq M}=\sum_{k=1}^{M} c_{k} \varphi_{k},
\end{array}
$$

respectively.

We call $\left\{\left\langle x, \varphi_{k}\right\rangle\right\}_{1 \leq k \leq M}$ the frame coefficients of $x$. It can be shown that $T^{*} T$ is invertible on $\mathbb{K}^{N}$ and, for each $x \in \mathbb{K}^{N}$,

$$
x=\sum_{k=1}^{M}\left\langle x, \varphi_{k}\right\rangle\left(T^{*} T\right)^{-1} \varphi_{k} .
$$

We call that $S=T^{*} T$ is the frame operator and $\left\{\widetilde{\varphi}_{k}=\right.$ $\left.\left(T^{*} T\right)^{-1} \varphi_{k}\right\}_{1 \leq k \leq M}$ the canonical dual frame for $\Phi$. It is easy to see that $S^{-1}$ is the frame operator of $\left\{\widetilde{\varphi}_{k}\right\}$. Generally, if $\left\{\psi_{k}\right\}_{1 \leq k \leq M}$ satisfies

$$
x=\sum_{k=1}^{M}\left\langle x, \varphi_{k}\right\rangle \psi_{k},
$$

for every $x \in \mathbb{K}^{N}$, we call that $\left\{\psi_{k}\right\}_{1 \leq k \leq M}$ is a dual frame for $\Phi$. For more details on frame theory and applications, we refer the reader to $[14,15]$ and so forth.

Fickus et al. gave the following definition.

Definition 6 (see [13, Definition 7]). A set of vectors $\left\{\varphi_{k}\right\}_{1 \leq k \leq M}$ in $\mathbb{K}^{N}$ gives almost phase retrieval if it gives phase retrieval for almost every $x \in \mathbb{K}^{N}$.

Next, we study real frames which give almost phase retrieval. Characterizing the complex frames which give almost phase retrieval remains an open problem.

Denote the $k$ th row and the $l$ th column of a matrix $A$ by $\operatorname{row}_{k} A$ and $\operatorname{col}_{l} A$, respectively. They gave a characterization of such frames.

Proposition 7 (see [13, Theorem 12]). Let $A$ be an $N \times M$ matrix on $\mathbb{R}$. Suppose each column of $A$ is nonzero. Then $A$ gives almost phase retrieval if and only if $A$ is of $\operatorname{rank} N$ and, for each nonempty proper subset $\Gamma \subset\{1,2, \ldots, M\}$,

$$
\operatorname{rank}(E)+\operatorname{rank}(F)>N,
$$

where $E$ and $F$ are the matrices consisting of $\left\{\operatorname{col}_{k} A\right\}_{k \in \Gamma}$ and $\left\{\operatorname{col}_{k} A\right\}_{k \in \Gamma^{C}}$, respectively.

Here we give another characterization which shows more properties of the almost phase retrieval. Let $\Gamma$ be a subset of $\{1,2, \ldots, M\}$. Denote

$$
\begin{gathered}
\alpha_{k}^{\Gamma}= \begin{cases}1, & k \in \Gamma \\
-1, & k \notin \Gamma,\end{cases} \\
k=1,2, \ldots, M .
\end{gathered}
$$

For a matrix $A$, denote

$$
A^{\Gamma}=A \cdot \operatorname{diag}\left(\alpha_{1}^{\Gamma}, \alpha_{2}^{\Gamma}, \ldots, \alpha_{M}^{\Gamma}\right) .
$$

Denote the range and null space of an operator or matrix $A$ by $\mathscr{R}(A)$ and $\mathscr{N}(A)$, respectively. Our first main result is the following.

Theorem 8. Let $\Phi=\left\{\varphi_{k}\right\}_{1 \leq k \leq M}$ be a frame for $\mathbb{R}^{N}$ with analysis operator $T$. Suppose that the $(M-N) \times M$ matrix A satisfies

$$
\mathcal{N}(A)=\mathscr{R}(T) .
$$

Then $\Phi$ gives almost phase retrieval if and only if

$$
\operatorname{rank}(A)<\operatorname{rank}\left(\begin{array}{c}
A \\
A^{\Gamma}
\end{array}\right)
$$

holds for every nonempty proper subset $\Gamma \subset\{1,2, \ldots, M\}$.

Proof. First we prove the necessity. We assume that there exists a nonempty proper subset $\Gamma \subset\{1,2, \ldots, M\}$ which does not satisfy (10). That is,

$$
\operatorname{rank}(A)=\operatorname{rank}\left(\begin{array}{c}
A \\
A^{\Gamma}
\end{array}\right)
$$


Since $\operatorname{rank}(A)=\operatorname{rank}\left(A^{\Gamma}\right)$, we have

$$
\mathcal{N}(A)=\mathcal{N}\left(A^{\Gamma}\right) .
$$

Hence for every

$$
c=\left\{c_{1}, c_{2}, \ldots, c_{M}\right\} \in \mathscr{R}(T),
$$

we have

$$
c^{\prime}=\left\{\alpha_{1}^{\Gamma} c_{1}, \alpha_{2}^{\Gamma} c_{2}, \ldots, \alpha_{M}^{\Gamma} c_{M}\right\} \in \mathscr{R}(T) .
$$

But $\alpha_{k}^{\Gamma} \neq \alpha_{l}^{\Gamma}$ for $k \in \Gamma$ and $l \in \Gamma^{C}$. Hence $\Phi$ does not give almost phase retrieval.

Sufficiency. Let $\Gamma$ be a nonempty proper subset of $\{1,2, \ldots$, $M$ \}. Then $\mathscr{N}(A) \cap \mathscr{N}\left(A^{\Gamma}\right)$ is a proper subspace of $\mathcal{N}(A)=$ $\mathscr{R}(T)$. Let $\mathscr{C}_{0}$ be the union of all such subspaces and $\mathcal{S}_{0}=T^{-1} \mathscr{C}_{0}$. Since $\mathcal{S}_{0}$ is the union of finitely many proper subspaces of $\mathbb{R}^{N}$, its Lebesgue measure in $\mathbb{R}^{N}$ is 0 . And for every $x \in \mathbb{R}^{N} \backslash \mathcal{S}_{0}, T x \notin \mathscr{C}_{0}$. That means $\Phi$ gives phase retrieval on $\mathbb{R}^{N} \backslash \mathcal{S}_{0}$. Hence $\Phi$ gives almost phase retrieval.

Remark 9. In Theorem 8, we only need $M>N$.

Proposition 7 can be considered as a consequence of Theorem 8. In fact, let $E$ and $F$ be defined as in Proposition 7. We have

$$
\begin{aligned}
\operatorname{rank}\left(\begin{array}{cc}
E & F \\
E & -F
\end{array}\right) & =\operatorname{rank}\left(\begin{array}{cc}
2 E & 0 \\
E & -F
\end{array}\right) \\
& =\operatorname{rank}\left(\begin{array}{cc}
2 E & 0 \\
0 & -F
\end{array}\right)=\operatorname{rank}(E)+\operatorname{rank}(F) .
\end{aligned}
$$

By Theorem 8, we get Proposition 7.

Now we have characterizations of frames which give almost phase retrieval. But it is tedious to test if a frame satisfies (6) or (10).

Next we give a method to test if a general frame gives almost phase retrieval. By Theorem 8, (11) holds if $\Phi$ does not give almost phase retrieval. First we give a condition for $A$ satisfying (11).

Lemma 10. Let $A$ be an $N \times M$ matrix on $\mathbb{R}$. Suppose that its columns are nonzero. Denote by $S$ the space spanned by rows of $A$. If the rows of $A$ are orthogonal basis of $S$, then, for every nonempty proper subset $\Gamma \subset\{1,2, \ldots, M\}$,

$$
\operatorname{rank}(A)=\operatorname{rank}\left(\begin{array}{c}
A \\
A^{\Gamma}
\end{array}\right)
$$

holds if and only if, for every $k \in \Gamma, l \in \Gamma^{C}$,

$$
\left\langle\operatorname{col}_{k} A, \operatorname{col}_{l} A\right\rangle=0 \text {. }
$$

Proof. First we prove the necessity. We assume that (16) holds. Then each row of $A^{\Gamma}$ is a linear combination of rows of $A$. Therefore there exists $c_{i j} \in \mathbb{R}$ such that

$$
\operatorname{row}_{k} A^{\Gamma}=c_{1 k} \operatorname{row}_{1} A+\cdots+c_{N k} \operatorname{row}_{N} A, \quad k=1,2, \ldots, N .
$$

That is,

$$
A^{\Gamma}=C A,
$$

where $C$ is an $N \times N$ matrix on $\mathbb{R}$. Since the rows of $A$ form an orthonormal basis of $S$, the rows of $A^{\Gamma}$ form also an orthonormal basis of $S$. Hence $C$ is an orthogonal matrix. Hence, for every $k \in \Gamma, l \in \Gamma^{c}$,

$$
\begin{aligned}
\left\langle\operatorname{col}_{k} A, \operatorname{col}_{l} A\right\rangle & =\left\langle C \cdot \operatorname{col}_{k} A, C \cdot \operatorname{col}_{l} A\right\rangle \\
& =\left\langle\operatorname{col}_{k} A^{\Gamma}, \operatorname{col}_{l} A^{\Gamma}\right\rangle=-\left\langle\operatorname{col}_{k} A, \operatorname{col}_{l} A\right\rangle .
\end{aligned}
$$

Therefore,

$$
\left\langle\operatorname{col}_{k} A, \operatorname{col}_{l} A\right\rangle=0 .
$$

Sufficiency. Denote by $E$ and $F$ the matrices consisting of $\left\{\operatorname{col}_{k} A\right\}_{k \in \Gamma}$ and $\left\{\operatorname{col}_{k} A\right\}_{k \in \Gamma^{C}}$, respectively. Let $S_{E}$ and $S_{F}$ be the spaces spanned by columns of $E$ and $F$, respectively. By (17), we have

$$
S_{E} \perp S_{F}
$$

Since each column of $A$ is nonzero, $S_{E}$ and $S_{F}$ are proper subspaces of $\mathbb{R}^{N}$. Therefore, there exists an $N \times N$ matrix $C$ on $\mathbb{R}$ such that, for every $x_{1} \in S_{E}$ and $x_{2} \in S_{F}$,

$$
C x_{1}=x_{1}, \quad C x_{2}=-x_{2} \text {. }
$$

Then we have

$$
C A=A^{\Gamma}
$$

Hence we get (16).

Lemma 10 shows that if the matrix $A$ can not be split into two orthogonal parts, then the corresponding frame $\Phi$ (see Theorem 8 ) gives almost phase retrieval. Here we need the fact that the rows of $A$ are orthogonal. If the columns of $A$ contain a natural basis, the result still holds.

Lemma 11. Let $A$ be an $N \times M$ matrix on $\mathbb{R}$. Suppose that the columns of A contain a natural basis $\left\{e_{i}\right\}_{1 \leq i \leq N}$ of $\mathbb{R}^{N}$. For every nonempty proper subset $\Gamma \subset\{1,2, \ldots, M\}$,

$$
\operatorname{rank}(A)=\operatorname{rank}\left(\begin{array}{c}
A \\
A^{\Gamma}
\end{array}\right)
$$

holds if and only if, for every $k \in \Gamma, l \in \Gamma^{c}$,

$$
\left\langle\operatorname{col}_{k} A, \operatorname{col}_{l} A\right\rangle=0 \text {. }
$$

Proof. For every nonempty proper subset $\Gamma \subset\{1,2, \ldots, M\}$, denote by $E$ and $F$ the matrices consisting of $\left\{\operatorname{col}_{k} A\right\}_{k \in \Gamma}$ and $\left\{\operatorname{col}_{k} A\right\}_{k \in \Gamma^{C}}$, respectively. Suppose that $E$ contains $\left\{e_{i}\right\}_{i \in \Theta}$ and $F$ contains $\left\{e_{i}\right\}_{i \in \Theta^{C}}$. Then,

$$
\left\langle\operatorname{col}_{k} A, \operatorname{col}_{l} A\right\rangle=0, \quad \forall k \in \Gamma, l \in \Gamma^{C},
$$

holds if and only if each column of $E$ is a linear combination of $\left\{e_{i}\right\}_{i \in \Theta}$ and each column of $F$ is a linear combination of 
$\left\{e_{i}\right\}_{i \in \Theta^{C}}$, which is equivalent to $\operatorname{rank}(E)=|\Theta|$ and $\operatorname{rank}(F)=$ $\left|\Theta^{C}\right|=N-\operatorname{rank}(E)$. Therefore,

$$
\operatorname{rank}\left(\begin{array}{c}
A \\
A^{\Gamma}
\end{array}\right)=\operatorname{rank}(E)+\operatorname{rank}(F)=N=\operatorname{rank}(A) .
$$

Next we show that we can choose a suitable matrix $A$ to test if $\Phi$ gives almost phase retrieval.

Theorem 12. Let $A$ be an $N \times M$ matrix on $\mathbb{R}$. Each column of $A$ is nonzero. Denote by $S$ the space spanned by rows of $A$. Let the $(M-N) \times M$ matrix $B$ consist of some $M-N$ linearly independent vectors in $S^{c} \subset \mathbb{R}^{M}$. If $A$ gives almost phase retrieval on $\mathbb{R}^{N}$, then $B$ gives almost phase retrieval on $\mathbb{R}^{M-N}$. Moreover, if rows of the $N \times M$ matrix $\widetilde{A}$ on $\mathbb{R}$ span $S$, then $\widetilde{A}$ also gives almost phase retrieval on $\mathbb{R}^{N}$.

Before proving Theorem 12, we introduce a result on the Naimark complement of Parseval frames.

Proposition 13 (Naimark's Theorem [14, Theorem 1.9]). Let $\Phi=\left\{\varphi_{k}\right\}_{1 \leq k \leq M}$ be a frame for $\mathbb{K}^{N}$ with analysis operator $T$. Let $\left\{e_{k}\right\}_{1 \leq k \leq M}$ be the standard basis of $l_{2}^{M}$, and let $P: l_{2}^{M} \rightarrow$ $l_{2}^{M}$ be the orthogonal projection onto $\mathscr{R}(T)$. Then the following conditions are equivalent.

(i) $\Phi$ is a Parseval frame for $\mathbb{K}^{N}$.

(ii) For all $k=1,2, \ldots, M$, we have $P e_{k}=T \phi_{k}$.

(iii) There exist $\left\{\psi_{k}\right\}_{1 \leq k \leq M} \subset \mathbb{K}^{M-N}$ such that $\left\{\varphi_{k} \oplus\right.$ $\left.\psi_{k}\right\}_{1 \leq k \leq M}$ is an orthonormal basis of $\mathbb{K}^{M}$.

Moreover, if (iii) holds, then $\left\{\psi_{k}\right\}_{1 \leq k \leq M}$ is a Parseval frame for $\mathbb{K}^{M-N}$.

We call that $\left\{\psi_{k}\right\}_{1 \leq k \leq M}$ is a Naimark complement of $\left\{\varphi_{k}\right\}_{1 \leq k \leq M}$.

Proof of Theorem 12. Take an $(M-N) \times M$ matrix $D$ whose rows are orthogonal basis of $S^{c}$. Then the columns of $D$ form a Parseval frame of $\mathbb{R}^{M-N}$. Since $\mathcal{N}(D)=\mathscr{R}(A)$ and $A$ gives almost phase retrieval, by Theorem 8 , for any nonempty proper subset $\Gamma \subset\{1,2, \ldots, M\}$, we have

$$
\operatorname{rank}(D)<\operatorname{rank}\left(\begin{array}{c}
D \\
D^{\Gamma}
\end{array}\right)
$$

By Lemma 10, there exist some $k \in \Gamma$ and $l \in \Gamma^{c}$ such that

$$
\left\langle\operatorname{col}_{k} D, \operatorname{col}_{l} D\right\rangle \neq 0 \text {. }
$$

Let $E$ be a Naimark complement of $D$. Then the rows of $E$ belong to $S$. Since

$$
\left\langle\operatorname{col}_{k} E, \operatorname{col}_{l} E\right\rangle+\left\langle\operatorname{col}_{k} D, \operatorname{col}_{l} D\right\rangle=0,
$$

we have

$$
\left\langle\operatorname{col}_{k} E, \operatorname{col}_{l} E\right\rangle \neq 0 \text {. }
$$

By Lemma 10,

$$
\operatorname{rank}(E)<\operatorname{rank}\left(\begin{array}{c}
E \\
E^{\Gamma}
\end{array}\right)
$$

Since $\mathcal{N}(E)=\mathscr{R}(B), B$ gives almost phase retrieval on $\mathbb{R}^{M-N}$, thanks to Theorem 8 .

From the above results, we give a method to test if an $N \times$ $M$ matrix $A$ gives almost phase retrieval on $\mathbb{R}^{N}$ or not.

Denote by $A_{1}$ and $A_{2}$ the matrices consisting of the first $N$ columns and the last $M-N$ columns of $A$, respectively. Without loss of generality, we assume that $A_{1}$ is of rank $N$. And $A_{1}^{-1} A$ has the following form:

$$
\widetilde{A}=\left(\begin{array}{ccccccc}
1 & 0 & \cdots & 0 & a_{1, N+1} & \cdots & a_{1, M} \\
0 & 1 & \cdots & 0 & a_{2, N+1} & \cdots & a_{2, M} \\
& & \cdots & & & \cdots & \\
0 & 0 & \cdots & 1 & a_{N, N+1} & \cdots & a_{N, M}
\end{array}\right)
$$

Then $\widetilde{A}$ gives almost phase retrieval if and only if $A$ does. Without loss of generality, we assume that each column of $\widetilde{A}$ is nonzero; otherwise we can drop the columns which are zero. Denote

$$
\operatorname{supp}\left(\operatorname{col}_{k} \widetilde{A}\right)=\left\{\lambda: \operatorname{col}_{k}^{\lambda} \widetilde{A} \neq 0\right\}
$$

where $\operatorname{col}_{k}^{\lambda} \widetilde{A}$ is the $\lambda$ th element of $\operatorname{col}_{k} \widetilde{A}$. Next we do the following steps.

(1) Set $k_{1}=N+1$. If $\operatorname{supp}\left(\operatorname{col}_{1} \widetilde{A}\right)=\{1,2, \ldots, N\}$, end. Otherwise, set

$\Gamma=\left\{k: \operatorname{supp}\left(\operatorname{col}_{k}\right) \subset \operatorname{supp}\left(\operatorname{col}_{k_{1}}\right), N+1 \leq k \leq M\right\}$.

If $\Gamma=\{N+1, N+2, \ldots, M\}$, end. Otherwise, go to step (2).

(2) Find a $k_{l} \in\{N+1, N+2, \ldots, M\} \backslash \Gamma$ such that $\operatorname{col}_{k_{l}}$ has two nonzero elements $\operatorname{col}_{k_{l}}^{\lambda_{1}} \widetilde{A}$ and $\operatorname{col}_{k_{l}}^{\lambda_{2}} \widetilde{A}$ satisfying $\lambda_{1} \in \bigcup_{1}^{l-1} \operatorname{supp}\left(\operatorname{col}_{k_{i}} \widetilde{A}\right)$ and $\lambda_{2} \epsilon$ $\left(\bigcup_{1}^{l-1} \operatorname{supp}\left(\operatorname{col}_{k_{i}} \widetilde{A}\right)\right)^{C}$. If there exists no such column of $\widetilde{A}$, end. Otherwise, go to step (3).

(3) If $\bigcup_{1}^{l} \operatorname{supp}\left(\operatorname{col}_{k_{i}} \widetilde{A}\right)=\{1,2, \ldots, N\}$, end. Otherwise, set

$\Gamma=\left\{k: \operatorname{supp}\left(\operatorname{col}_{k}\right) \subset \bigcup_{1}^{l} \operatorname{supp}\left(\operatorname{col}_{k_{i}} \widetilde{A}\right), N+1 \leq k \leq M\right\}$.

If $\Gamma=\{N+1, N+2, \ldots, M\}$, end. Otherwise, go to step (2).

After finitely many steps, say $L$ steps, we get $\Gamma$ and $\bigcup_{1}^{L} \operatorname{supp}\left(\operatorname{col}_{k_{i}} \widetilde{A}\right) \triangleq \Lambda$ finally. There are two cases. 
(i) Consider $\Lambda \neq\{1,2, \ldots, N\}$. For every $k_{1} \in \Gamma \cup \Lambda$ and $k_{2} \in(\{N+1, N+2, \ldots, M\} \backslash \Gamma) \cup \Lambda^{C}=\{1,2, \ldots, M\} \backslash$ $(\Gamma \cup \Lambda)$, we have

$$
\left\langle\operatorname{col}_{k_{1}} \widetilde{A}, \operatorname{col}_{k_{2}} \widetilde{A}\right\rangle=0
$$

By Lemma 11 and Theorem $8, \widetilde{A}$ does not give almost phase retrieval and neither does $A$.

(ii) Consider $\Lambda=\{1,2, \ldots, N\}$. For any $k_{1}, k_{2} \in$ $\{1,2, \ldots, N\}$, there exist $j_{1}, j_{2}, \ldots, j_{s} \in\{1,2, \ldots, M\}$ such that

$$
\begin{gathered}
\left\langle\operatorname{col}_{k_{1}} A, \operatorname{col}_{j_{1}} A\right\rangle \neq 0, \\
\left\langle\operatorname{col}_{j_{1}} A, \operatorname{col}_{j_{2}} A\right\rangle \neq 0, \\
\vdots \\
\left\langle\operatorname{col}_{j_{s-1}} A, \operatorname{col}_{j_{s}} A\right\rangle \neq 0, \\
\left\langle\operatorname{col}_{j_{s}} A, \operatorname{col}_{k_{2}} A\right\rangle \neq 0 .
\end{gathered}
$$

Therefore, there exists some nonempty proper subset $\widetilde{\Gamma} \subset\{1,2, \ldots, M\}$ satisfying (26). Hence $\widetilde{A}$ gives almost phase retrieval, and so does $A$.

Remark 14. Balan et al. [4, Theorem 2.9] proved that $\widetilde{A}$ gives almost phase retrieval while $\widetilde{A}$ has a column $\operatorname{col}_{k} A$ such that supp $\operatorname{col}_{k} A=\{1,2, \ldots, M\}$. By the above method, it is easy to see that $\widetilde{A}$ gives almost phase retrieval. And we find more general matrices which give almost phase retrieval in some sense.

Remark 15. In our method, we need to compute the inverse of $A_{1}$. But $\operatorname{rank}(A)=N$ is necessary; the processes of transforming $A$ to $\widetilde{A}$ and computing $\operatorname{rank}(A)$ are conducted at the same time. Next it is very easy to compute $\Gamma$. Therefore, the computation complexity $O(M-N)$ is acceptable.

Here we give a method to test if a frame gives almost phase retrieval or not. With this method, we can construct frames which have this property. For the unit norm tight frames, Fickus et al. [13] gave a sufficient condition.

Proposition 16 (see [13, Theorem 13]). If $M$ and $N$ are relatively prime, then every unit norm tight frame $\Phi=$ $\left\{\varphi_{k}\right\}_{1 \leq k \leq M} \in \mathbb{R}^{N}$ gives almost phase retrieval.

Different from Proposition 16, for any $M>N$, we can construct a tight frame $\Phi=\left\{\phi_{k}\right\}_{1 \leq k \leq M}$ which gives almost phase retrieval from the above methods.

First, we can construct an $N \times M$ matrix $\widetilde{A}$ of the form (34) such that it gives almost phase retrieval. For example, we can choose $\widetilde{A}$ such that one of the last $M-N$ columns does not contain zero element. Second, by the Gram-Schmidt process or other methods, we get a matrix $\widehat{A}$ from $\widetilde{A}$ such that the rows of $\widehat{A}$ are orthogonal. Third, let

$$
\widetilde{A}=C \cdot\left(\begin{array}{c}
\frac{\operatorname{row}_{1}(\widehat{A})}{\left\|\operatorname{row}_{1}(\widehat{A})\right\|_{2}} \\
\frac{\operatorname{row}_{2}(\widehat{A})}{\left\|\operatorname{row}_{2}(\widehat{A})\right\|_{2}} \\
\vdots \\
\frac{\operatorname{row}_{N}(\widehat{A})}{\left\|\operatorname{row}_{N}(\widehat{A})\right\|_{2}}
\end{array}\right),
$$

where $C \in \mathbb{R}$ is a constant. Then the columns of $A$ form a tight frame with frame bound $C$ by the following proposition.

Proposition 17 (see [16, Lemma 1.1]). The columns of an $N \times$ $M$ matrix $A$ are a tight frame with frame bound $C$ if and only if

$$
A \cdot A^{T}=C \cdot I d .
$$

If an $N \times M$ matrix $A$ does not give almost phase retrieval, we know that $\Lambda \neq\{1,2, \ldots, N\}$ from the above method. Then we can choose a column of $\widetilde{A}$, say $\operatorname{col}_{k_{0}} \widetilde{A}$, such that $k_{0} \in\{N+1, N+2, \ldots, M\}$ and $\operatorname{supp}\left(\operatorname{col}_{k_{0}}\right) \subset \Lambda$. Let $\widetilde{B}$ be a matrix satisfying

$$
\operatorname{col}_{k} \widetilde{B}= \begin{cases}\operatorname{col}_{k} \widetilde{A}, & k \neq k_{0}, \\ \operatorname{col}_{k} \widetilde{A}+v, & k=k_{0},\end{cases}
$$

where

$$
v_{j}= \begin{cases}\epsilon_{j} \neq 0, & j \in \Lambda^{C}, \\ 0, & \text { others. }\end{cases}
$$

Then we can check that set $\Lambda$ with respect to $\widetilde{B}$ is $\{1,2, \ldots, N\}$. Let $B=A_{1} \widetilde{B}$. Then $B$ gives almost phase retrieval. On the other hand, the canonical dual frame is always used to reconstruct the original vector. Sun [17] gave the following stability result of canonical dual frame.

Proposition 18 (see [17, Theorem 4.1]). Let $\Phi$ and $\widetilde{\Phi}$ and $\Psi$ and $\widetilde{\Psi}$ be two pairs of canonical dual frames for $\mathbb{R}^{N}$. Denote the frame operator of $\Phi$ and $\Psi$ by $S$ and $W$, respectively. Denote the frame bounds of $\Phi$ and $\Psi$ by $\left(\alpha_{1}, \beta_{1}\right)$ and $\left(\alpha_{2}, \beta_{2}\right)$, respectively. Then

(i) if $\Phi-\Psi$ is a Bessel sequence with an upper bound $\delta$, then so is $\widetilde{\Phi}-\widetilde{\Psi}$ with an upper bound $\delta\left(\left(\alpha_{1}+\beta_{1}+\right.\right.$ $\left.\left.\beta_{1}^{1 / 2} \beta_{2}^{1 / 2}\right) /\left(\alpha_{1} \alpha_{2}\right)\right)^{2}$.

(ii) If

$$
|\langle S f, f\rangle-\langle W f, f\rangle| \leq \delta\|f\|_{2}^{2},
$$

then

$$
\left|\left\langle S^{-1} f, f\right\rangle-\left\langle W^{-1} f, f\right\rangle\right| \leq \frac{\delta}{\alpha_{1} \alpha_{2}}\|f\|_{2}^{2} .
$$




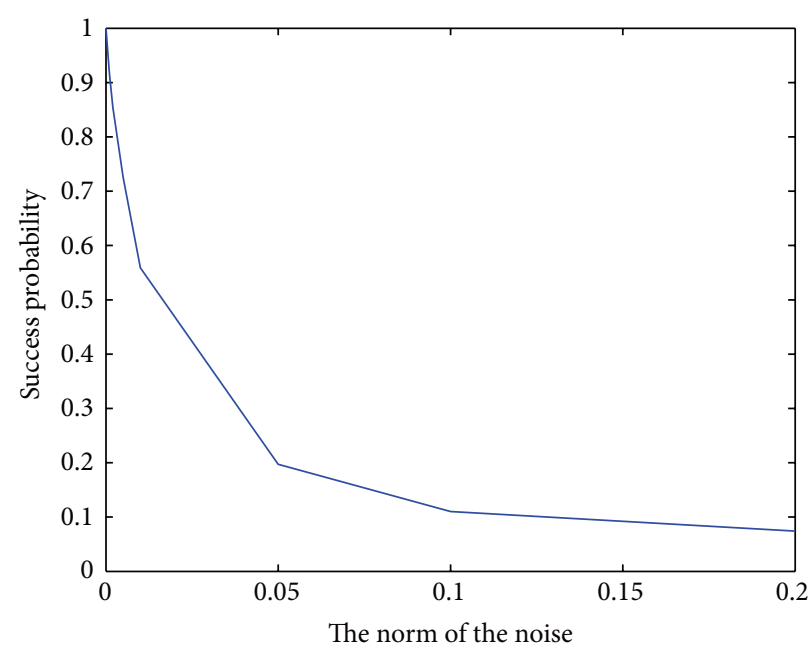

(a) Trial 1

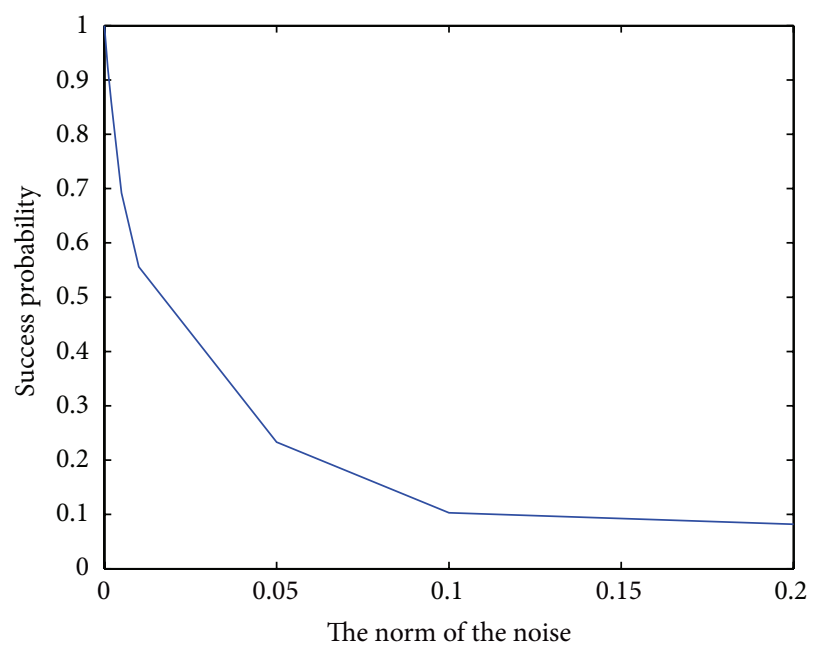

(c) Trial 3

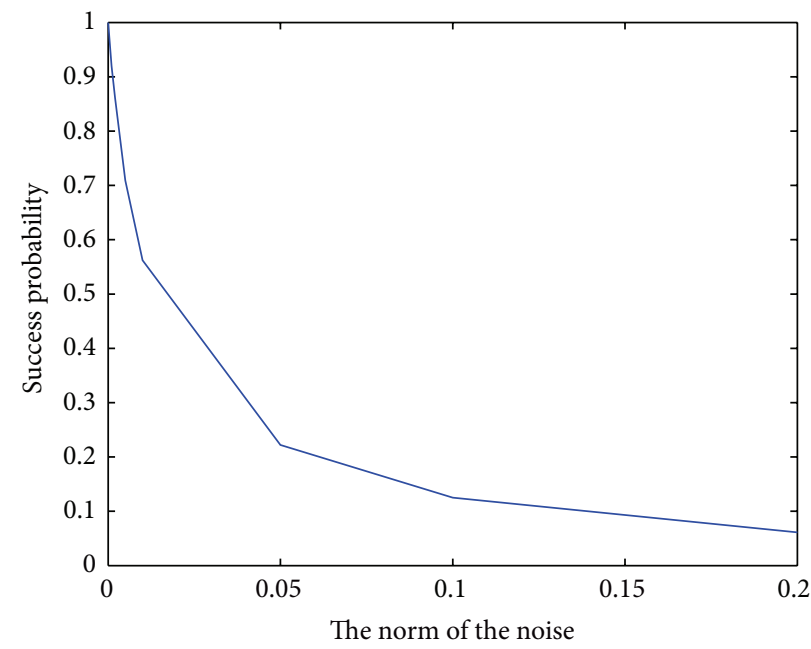

(b) Trial 2

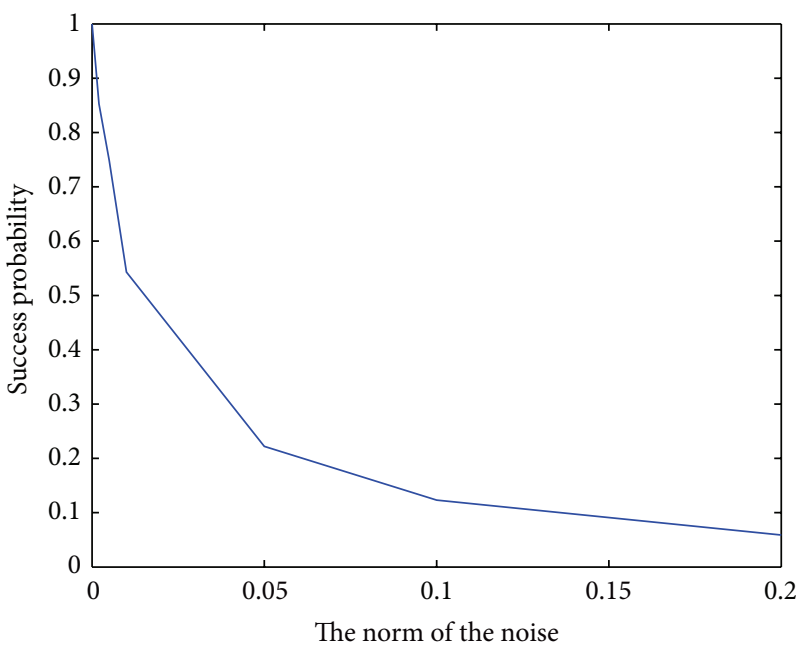

(d) Trial 4

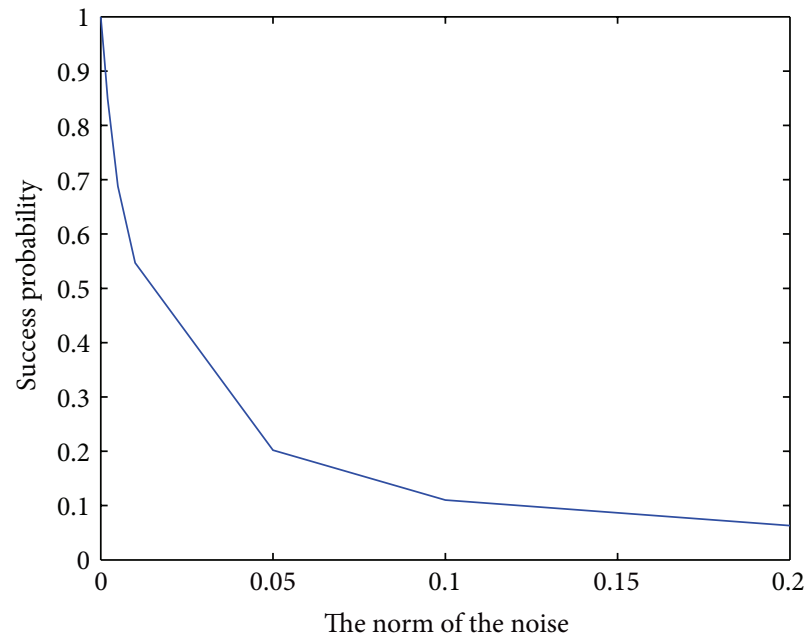

(e) Trial 5

FIGURE 1: Recovery of signals from their intensity measurements with different noise levels, where $N=8$ and $M=9$. 


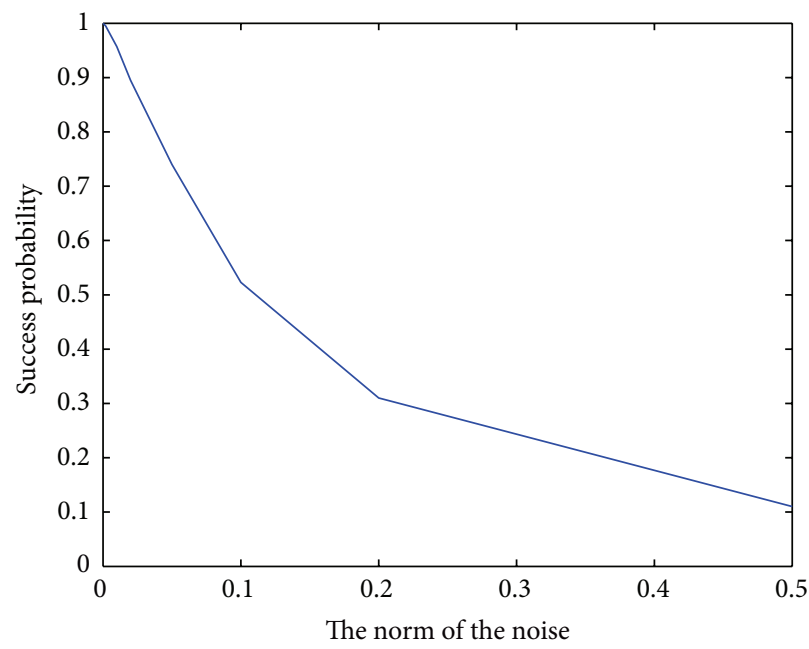

(a) Trial 1

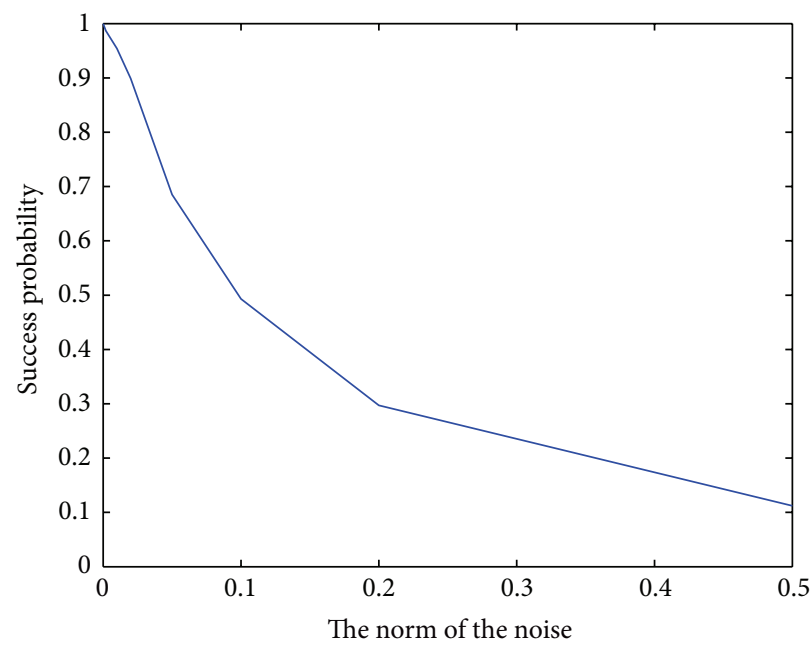

(c) Trial 3

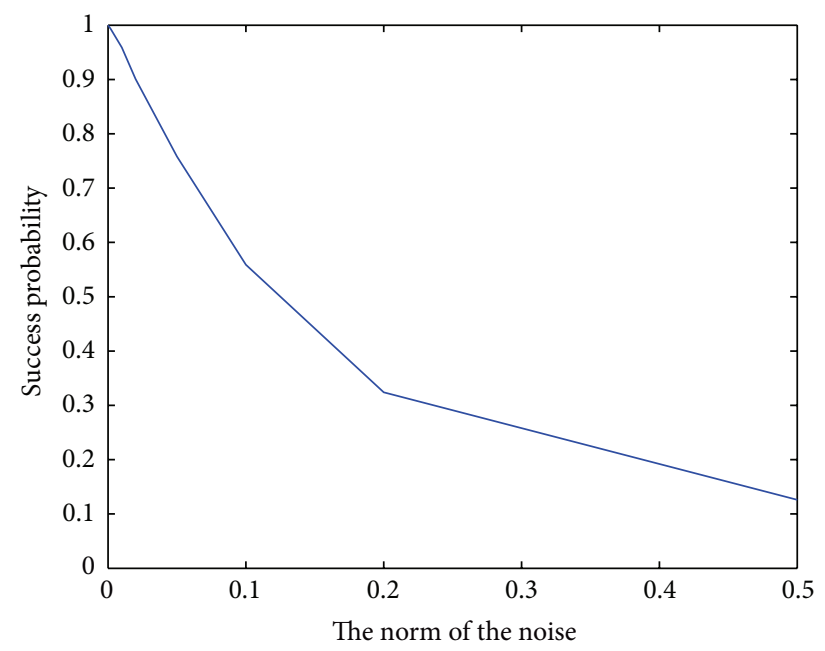

(b) Trial 2

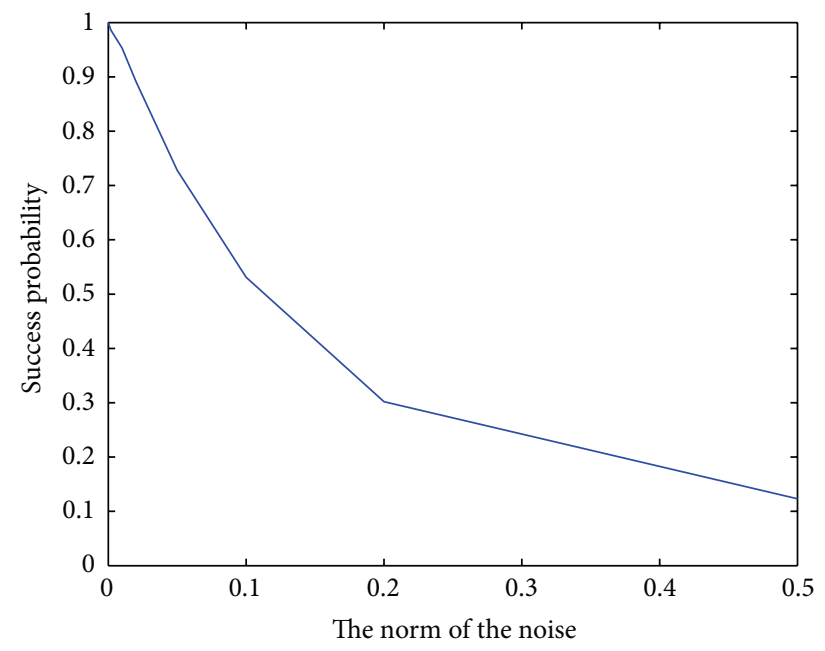

(d) Trial 4

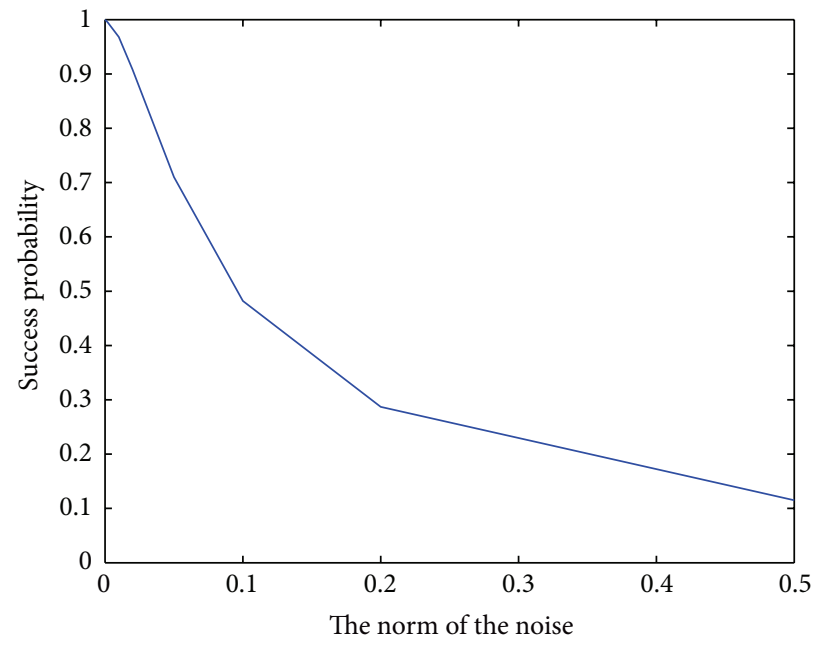

(e) Trial 5

FIGURE 2: Recovery of signals from their intensity measurements with different noise levels, where $N=8$ and $M=10$. 
Since

$$
\begin{aligned}
\|(B-A) x\|_{2}^{2} & \leq\left\|A_{1}\right\|_{2}^{2}\|(\widetilde{B}-\widetilde{A}) x\|_{2}^{2} \\
& =\left\|A_{1}\right\|_{2}^{2}|\langle x, v\rangle|^{2} \leq\|v\|_{2}^{2}\left\|A_{1}\right\|_{2}^{2}\|x\|_{2}^{2},
\end{aligned}
$$

for every $x \in \mathbb{R}^{N}$, for $\|v\|_{2}^{2}$ small enough, the matrix $B$ is a good approach of $A$. Denote the frames consisting of the columns of $A$ and $B$ by $\Phi$ and $\Psi$, respectively. From Proposition 18, we see that the canonical dual frame of $\Psi$ is also a good approach of the canonical dual frame of $\Phi$. Hence, if $A$ does not give almost phase retrieval, we can get a new matrix $B$ which gives almost phase retrieval with a little change. And the process system is stable.

\section{Numerical Results}

In this section, we provide some examples to show the frames in Theorem 8 give almost phase retrieval.

Let $\Phi=\left\{\varphi_{k}\right\}_{1 \leq k \leq M}$ be a frame for $\mathbb{R}^{N}$ with analysis operator $T$. For $x \in \mathbb{R}^{N}$, we want to recover $x$ from $\left\{\left|\left\langle x, \varphi_{k}\right\rangle\right|\right\}_{1 \leq k \leq M}$. Based on Theorem 8, we use the following algorithm.

(i) Take some $(M-N) \times M$ matrix $A$ such that $\mathcal{N}(A)=$ $\mathscr{R}(T)$. For every $\alpha=\left(\alpha_{1}, \alpha_{2}, \ldots, \alpha_{M}\right), \alpha_{k}= \pm 1,1 \leq$ $k \leq M$, denote

$$
b_{\alpha}=\left(\alpha_{1}\left|\left\langle x, \varphi_{1}\right\rangle\right|, \alpha_{2}\left|\left\langle x, \varphi_{2}\right\rangle\right|, \ldots,\left|\left\langle x, \alpha_{M} \varphi_{M}\right\rangle\right|\right) .
$$

(ii) Compute $\epsilon_{\alpha}=A \cdot b_{\alpha}$. Set

$$
\alpha^{0}=\underset{\alpha}{\operatorname{argmin}}\left\|A \cdot b_{\alpha}\right\|_{2}
$$

(iii) Set $y=\sum_{1 \leq k \leq M} \alpha_{k}^{0}\left|\left\langle x, \varphi_{k}\right\rangle\right| \psi_{k}$, where $\left\{\psi_{k}\right\}_{1 \leq k \leq M}$ is a dual frame of $\Phi$. Then $y= \pm x$.

A practical method to construct the desired frame $\Phi$ is using random vectors. Given $M$ and $N$, let $\Phi$ be a set of vectors in $\mathbb{R}^{N}$ with entries generated with the Gaussian random variable. In our tests, any frame generated in this way satisfies $(10)$. For simplicity, we take $(N, M)=(8,9)$ and $(N, M)=(8,10)$.

We test our algorithm to recover signals from their intensity measurements with different noise levels. That is, the measurement is $\left\{\left|\left\langle x, \varphi_{k}\right\rangle\right|+n_{k}\right\}_{1 \leq k \leq M}$ where $n=$ $\left(n_{1}, n_{2}, \ldots, n_{M}\right)$ is a noise with the norm $\sigma$. We perform 5 trials. In each trial, we generate a set of new random vectors and solve the phase retrieval problem with 1000 random vectors with norm 1 . Figures 1 and 2 show the results of the 5 trials. We see that the result is perfect and noise free. Whenever $\sigma<1 / 2^{M-1}$, the performance is good. Observe that the aliasing occurs for signals in $S_{0}$ consisting of $2^{M-1}$ proper subspaces. The results are also acceptable with larger noises. This shows that the frames in Theorem 8 can give phase retrieval with high probability.

\section{Conclusion}

In this paper, we show that $N+1$ intensity measurements are enough to reconstruct almost all signals of length $N$. With our characterization, we provide a method to test if a frame has this property. Numerical results show that we can use these frames to recover signals with high probability.

\section{Conflict of Interests}

The author declares that there is no conflict of interests regarding the publication of this paper.

\section{References}

[1] C. Becchetti and K. P. Ricotti, Speech Recognition: Theory and C++ Implementation(With CD), John Wiley \& Sons, 2008.

[2] M. Dierolf, A. Menzel, P. Thibault et al., "Ptychographic X-ray computed tomography at the nanoscale," Nature, vol. 467, no. 7314, pp. 436-439, 2010.

[3] P. G. Casazza and L. M. Woodland, "Phase retrieval by vectors and projections".

[4] R. Balan, P. Casazza, and D. Edidin, "On signal reconstruction without phase," Applied and Computational Harmonic Analysis, vol. 20, no. 3, pp. 345-356, 2006.

[5] A. Conca, D. Edidin, M. Hering, and C. Vinzant, "An algebraic characterization of injectivity in phase retrieval," http://arxiv .org/abs/1312.0158.

[6] A. S. Bandeira, J. Cahill, D. G. Mixon, and A. A. Nelson, "Saving phase: injectivity and stability for phase retrieval," Applied and Computational Harmonic Analysis, vol. 37, no. 1, pp. 106-125, 2014.

[7] H. Ohlsson and Y. C. Eldar, "On conditions for uniqueness in sparse phase retrieval," http://arxiv.org/abs/1308.5447.

[8] Y. C. Eldar and S. Mendelson, "Phase retrieval: stability and recovery guarantees," Applied and Computational Harmonic Analysis, vol. 36, no. 3, pp. 473-494, 2014.

[9] D. Gross, F. Krahmer, and R. Kueng, "A partial der andomization of phaselift using spherical designs," http://arxiv.org/abs/ 1310.2267 .

[10] B. Alexeev, A. S. Bandeira, M. Fickus, and D. G. Mixon, "Phase retrieval with polarization," SIAM Journal on Imaging Sciences, vol. 7, no. 1, pp. 35-66, 2014.

[11] Y. Chen, Y. Chi, and A. Goldsmith, "Exact and st able covariance estimation from quadratic sampling via convex programming," 2013, http://arxiv.org/abs/1310.0807.

[12] P. Schniter and S. Rangan, "Compressive phase retrieval via generalized approximate message passing," in Proceedings of the 50th IEEE Annual Allerton Conference on Communication, Control, and Computing, pp. 815-822, Allerton, Iowa, USA, 2012.

[13] M. Fickus, D. G. Mixon, A. A. Nelson, and Y. Wang, "Phase retrieval from very few measurements," Linear Algebra and Its Applications, vol. 449, pp. 475-499, 2014.

[14] P. G. Casazza and G. Kutyniok, Finite Frames: Theory and Applications, Springer, 2013.

[15] O. Christensen, An Introduction to Frames and Riesz Bases, Springer, 2003. 
[16] G. Zimmermann, "Normalized tight frames in finite dimensions," in Recent Progress in Multivariate Approximation, pp. 249-252, Springer, 2001.

[17] W. Sun, "Stability of $g$-frames," Journal of Mathematical Analysis and Applications, vol. 326, no. 2, pp. 858-868, 2007. 


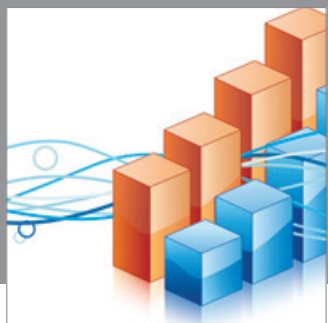

Advances in

Operations Research

mansans

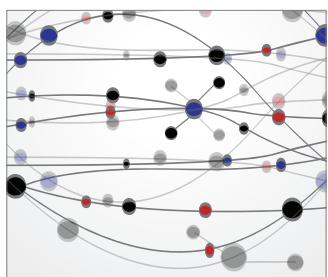

The Scientific World Journal
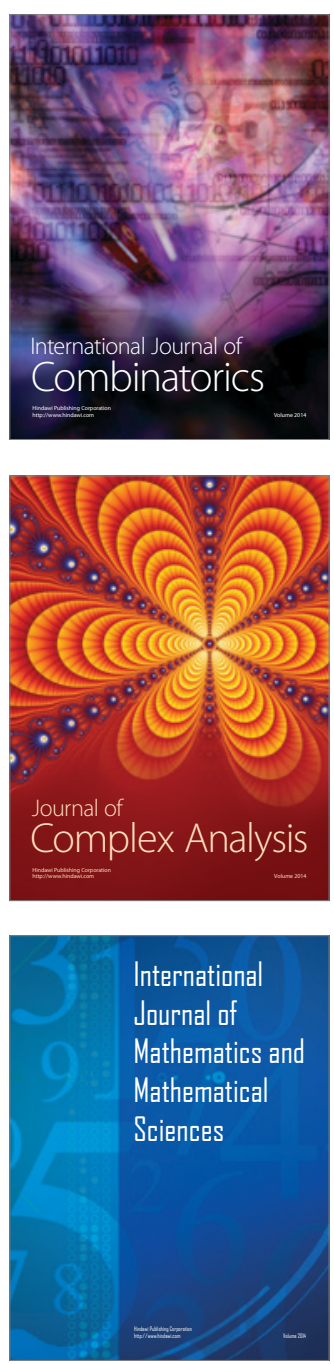
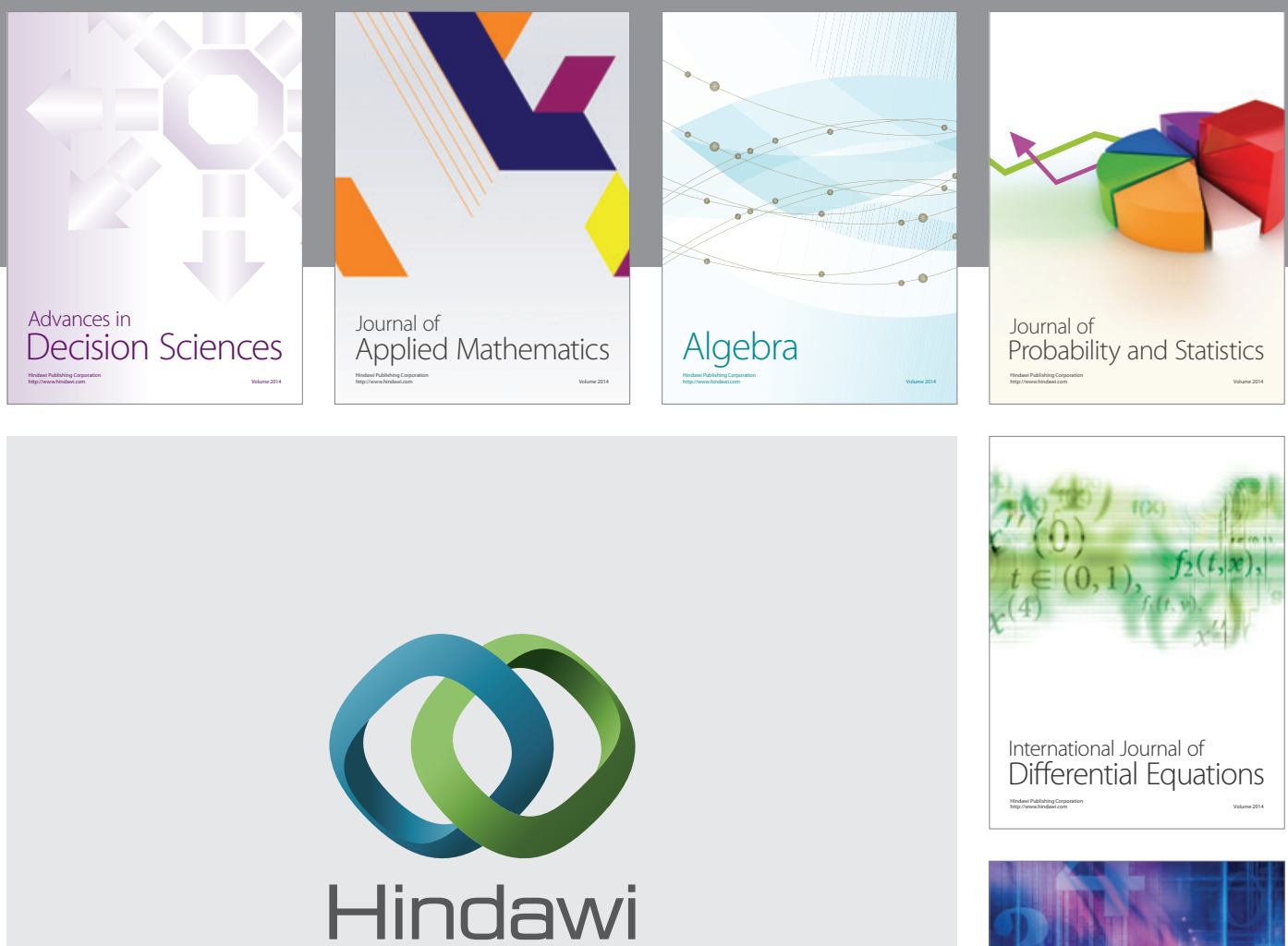

Submit your manuscripts at http://www.hindawi.com
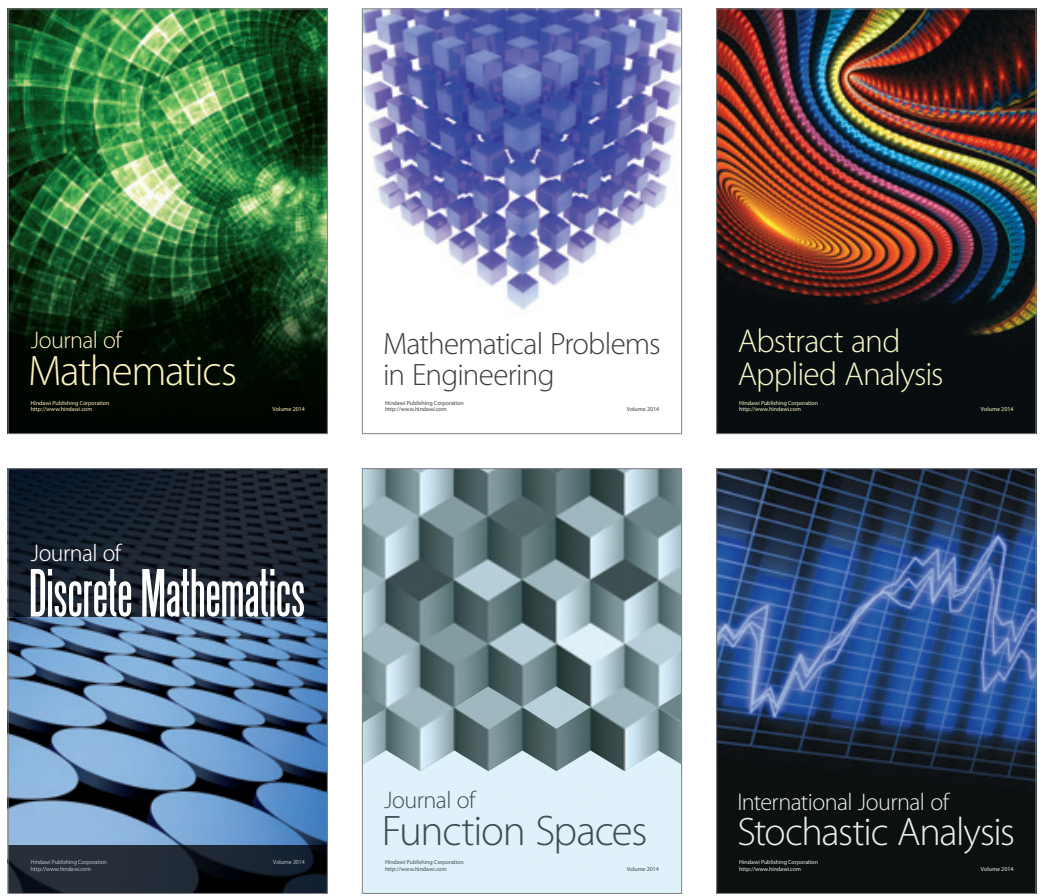

Journal of

Function Spaces

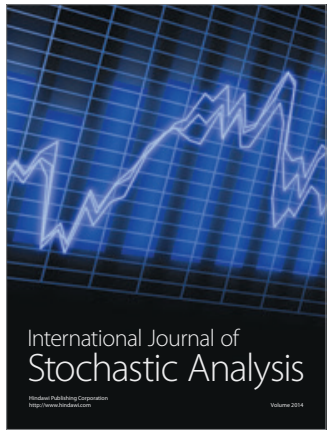

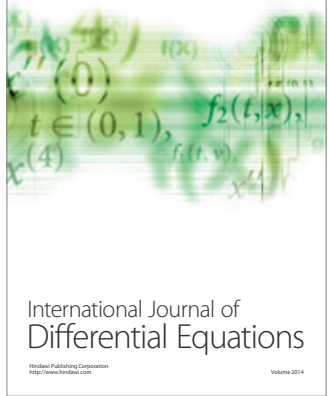
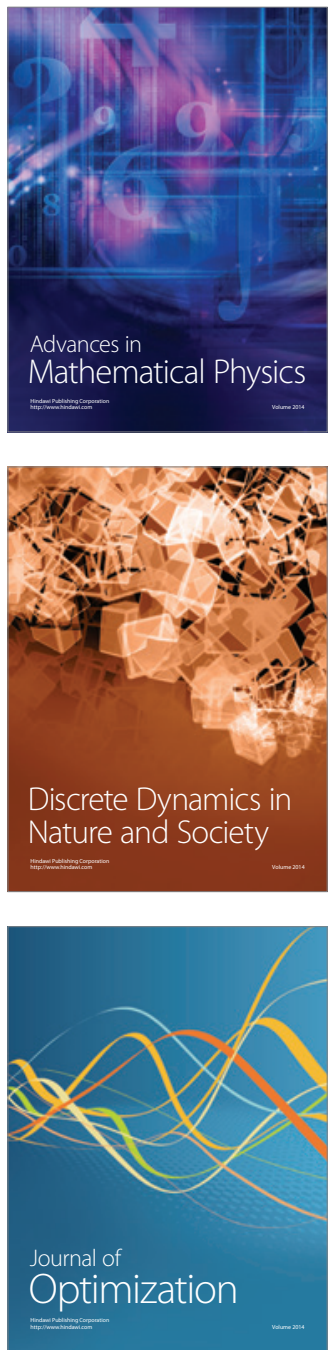